\title{
THE
}

\section{Brazilian Immigrant Mothers' Beliefs and Practices Related to Infant Feeding: A Qualitative Study}

Ana Cristina Lindsay

Sherrie F. Wallington

Mary L. Greaney

University of Rhode Island, mgreaney@uri.edu

Maria Helena Hasselman

Marcia M. T. Machado

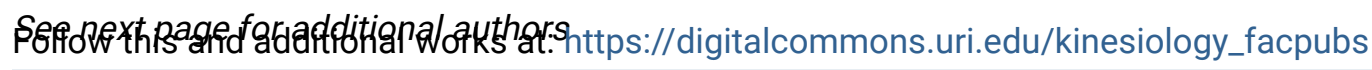

The University of Rhode Island Faculty have made this article openly available.

Please let us know how Open Access to this research benefits you.

This is a pre-publication author manuscript of the final, published article.

Terms of Use

This article is made available under the terms and conditions applicable towards Open Access

Policy Articles, as set forth in our Terms of Use.

\section{Citation/Publisher Attribution}

Lindsay, A. C., Wallington, S. F., Greaney, M. L., Hasselman, M. H., Machado M. M.T., \& Mezzavilla, R. S. (2017). Brazilian Immigrant Mothers' Beliefs and Practices Related to Infant Feeding: A Qualitative Study. Journal of Human Lactation, 33(3), 595-605. doi: 10.1177/0890334416676267

Available at: https://doi.org/10.1177/0890334416676267

This Article is brought to you for free and open access by the Kinesiology at DigitalCommons@URI. It has been accepted for inclusion in Kinesiology Faculty Publications by an authorized administrator of DigitalCommons@URI. For more information, please contact digitalcommons-group@uri.edu. 


\section{Authors}

Ana Cristina Lindsay, Sherrie F. Wallington, Mary L. Greaney, Maria Helena Hasselman, Marcia M. T. Machado, and Raquel S. Mezzavilla 


\section{Brazilian Immigrant Mothers' Beliefs and Practices Related to Infant Feeding: A}

\section{Qualitative Study}

Ana Cristina Lindsay, DDS, MPH, DrPH—University of Massachusetts Boston: Exercise and

Health Sciences Department—College of Nursing and Health Sciences, Boston, MA.

Sherrie F. Wallington, MS, PhD_Lombardi Comprehensive Cancer Center, Georgetown

University Medical Center, Washington, DC.

Mary L. Greaney, MS, PhD_ University of Rhode Island: Health Studies \& Department of

Kinesiology, Kingston, RI.

Maria Helena Hasselman, RD, MS, PhD—Rio de Janeiro State University: Department of Social

Nutrition, Rio de Janeiro, Brazil.

Marcia Maria Tavares Machado, RN, MS, PhD— Federal University of Ceara: School of

Medicine, Department of Community Health, Fortaleza, Ceara, Brazil.

Raquel S. Mezzavilla, RD, MS, PhD—Rio de Janeiro State University: Department of Social

Nutrition, Rio de Janeiro, Brazil.

Address for Correspondence:

Ana Cristina Lindsay, DDS, MPH, DrPH, Associate Professor

Exercise and Health Sciences Department

College of Nursing and Health Sciences

University of Massachusetts Boston

100 Morrissey Boulevard

Boston, MA 02125

Phone: 617-287-7579

Email: Ana.Lindsay@umb.edu 


\section{Acknowledgments}

We are grateful for help from personnel at Saint Anthony Churches in Brighton and Somerville and Igreja Evangelica Louvai of Framingham, Massachusetts. Ms. Mezzavilla received a scholarship from the Brazilian National Council for Research and Development. Research was supported by The Joseph Healey Grant, University of Massachusetts Boston (Lindsay, PI).

\section{Well-Established}

Length of stay in the United States is associated with decreased initiation and reduced duration of breastfeeding among Latina immigrants. The longer the length of residence, the less likely women are to initiate breastfeeding and/or maintain breastfeeding.

\section{Newly-Expressed}

This is the first research to examine beliefs and infant feeding practices of Brazilian immigrant mothers living in United States. This research is needed to identify risk factors amenable to interventions culturally tailored to the specific needs of this ethnic group. 


\begin{abstract}

\section{Background}

Exclusive breastfeeding for the first six months of life and timely introduction of appropriate solid foods are important determinants of weight status in infancy and later life stages. Disparities in obesity rates among young children suggest that maternal feeding practices during the first two years of life may contribute to these disparities. Brazilians are a growing immigrant group in the United States, yet little research has focused on parental beliefs and behaviors affecting health of Brazilian immigrant children in the United States.

\section{Research Aim}

Explore beliefs and infant feeding practices of Brazilian immigrant mothers in the United States.

\title{
Methods
}

Focus group discussions were conducted with Brazilian immigrant mothers. Transcripts were analyzed using thematic analysis and themes categorized using the socioecological model.

\section{Results}

Twenty-nine immigrant Brazilian mothers participated in the study. Analyses revealed all participants breastfed their infants. The majority initiated breastfeeding soon after childbirth. However, most mothers did not exclusively breastfeed. They used formula and breast milk concomitantly. Family and culture influenced mothers' infant feeding beliefs and practices early introduction of solid foods.

\section{Conclusions}

As the number of children in the United States growing up in families of immigrant parents increases, understanding influences on Brazilian immigrant mothers' infant feeding practices will be important to development of effective interventions to promote healthy infant 
feeding and weight status among Brazilian children. Interventions designed for Brazilian immigrant families should incorporate an understanding of social context, family and cultural factors to develop health promotion messages tailored to the needs of this ethnic group. 


\section{Background}

Infancy is recognized as a critical period of growth and development that influences children's health with long-lasting consequences (Baker, Michaelsen, Rasmussen, \& Sorensen, 2004; Institute of Medicine, Food and Nutrition Board, 2015; Fewtrell, Haschke, \& Prescott, 2014). Therefore, identifying modifiable risk factors amenable to intervention during this life stage is of central importance in the prevention of childhood obesity and its associated comorbidities (Institute of Medicine, 2011; World Health Organization, 2014; Lumeng, Taveras, Birch, \& Yanovski, 2015). Approximately $8.1 \%$ of U.S. children younger than age 2 years have weight-for-length $\geq 95$ th percentile, increasing their risk of obesity (Ogden, Carroll, Kit, \& Flegal, 2014; Pan, Grummer-Strawn, McGuire, Park, \& Blanck, 2015). Racial and ethnic minority children of low-income immigrant families are at increased risk of obesity in the first two years of life (Kimbro, Brooks-Gunn, \& McLanahan, 2007; Sanchez-Vaznaugh, Kawachi, Subramanian, Sanchez, \& Acevedo-Garcia, 2008; Ebenegger et al., 2011; Singh, Siahpush, Hiatt, \& Timsina, 2011; Dixon, Pena, \& Taveras, 2012; Ogden et al., 2014; Pan et al., 2015).

The American Academy of Pediatrics recommends exclusive breastfeeding (EBF) infants until six months of age, then continuing breastfeeding until at least one year of age and introducing nutritious solid foods at about six months of age (American Academy of Pediatrics, 2012). In addition, the World Health Organization (WHO) recommends that women breastfeed their infants for 2+ years (2008). Nonetheless, the worldwide prevalence of EBF is low and early introduction of complementary feeding is high (World Health Organization). Studies conducted with Latina immigrants living in the United States suggest that breastfeeding initiation and length of breastfeeding among Latina women varies according to cultural factors such as country of origin and years living in the United States (Sussner, Lindsay, \& Peterson, 2008; Verstraete, Heyman, \& Wojcicki, 2014). Higher levels of acculturation are associated with lower rates of 
breastfeeding initiation and shorter duration of breastfeeding (Sussner et al., 2008; Verstraete et al., 2014). The most recent national survey conducted in Brazil determined that, $95 \%$ of mothers initiate breastfeeding, but that the prevalence of EBF, which varies across regions, is low (Brasil, Ministerio da Saude, Centro Brasileiro de Analise e Planejamento, 2009). Furthermore, studies conducted in Brazil show that the number of women who adhere to WHO breastfeeding recommendation is low. Only about half of lactating women maintain breastfeeding up to 12 months and only one-fourth continue for two years (Brasil, Ministerio da Saude, Centro Brasileiro de Analise e Planejamento, 2009; Venancio, Escuder, Saldiva, \& Giugliani, 2010; Vieira et al., 2014).

Early feeding practices, including non-exclusive and short duration of breastfeeding $(<4$ months), and infant feeding practices such as introducing solid foods early, serving juices, and adding cereal to the bottle are associated with increased risk of obesity in infancy and early childhood, which may carry into later life stages (Weng, Redsell, Swift, Yang, \& Glazebrook, 2012; Pearce, Taylor, \& Langley-Evans, 2013; Thompson \& Bentley, 2013; Gross, Mendelsohn, Fierman, Hauser, \& Messito, 2014; Yan, Liu, Zhu, Huang, \& Wang, 2014).

Brazilians are a fast-growing immigrant group in the United States, yet very little research has focused on health issues affecting Brazilian immigrant women and their children living in the United States (Lima \& Siqueira, 2007; Godfrey, Jouet-Pastre, \& Praga, 2009). According to data from the 2006-2010 American Community Survey of approximately 400,000 Brazilian immigrants living in the United States, nearly half live in the northeastern states, mostly in Massachusetts (MA), New York, and New Jersey (Lima \& Siqueira, 2007). A recent study conducted in the Greater Boston area, MA, found that $48.2 \%$ of Brazilian immigrant children (aged 3-12 years) were overweight or obese (Tovar et al., 2013). Minimizing obesity disparities in early childhood requires a greater understanding of early maternal-infant feeding 
(Dattilo et al., 2012). To our knowledge, no existing research has examined infant feeding beliefs and practices of Brazilian immigrant mothers living in the United States. This information is needed to identify risk factors amenable to interventions and to design culturally appropriate interventions tailored to the specific needs of this ethnic group. Given the increased risk of early childhood obesity in immigrant and minority children and the potential influence of early feeding practices on weight, the aim of this investigation was to explore beliefs about infant feeding and practices such as breastfeeding and the process of complementary feeding of Brazilian-born immigrant mothers living in the United States.

\section{Methods}

We used the socioecological model (SEM) to provide a systematic consideration of personal, family, environmental, and organizational influences on mothers' beliefs, attitudes, and practices related to infant feeding amenable to intervention and modification (McLeroy, Bibeau, Steckler, \& Glanz, 1988). The SEM was also used to develop the moderator guide for the focus group discussions (FGDs) for this study, and to categorize emergent themes by levels: (a) individual (e.g., beliefs, attitudes, practices, daily hassles); (b) interpersonal (e.g., family, friends, healthcare providers, social support, social networks); (c) environmental (e.g., exposure to different physical environments), and (d) organizational influences (e.g., healthcare programs) (McLeroy et al., 1988).

\section{Design}

Focus group discussions were conducted with Brazilian immigrant mothers. We conducted focus group discussions (FDGs) because they are an important technique for conducting research in diverse cultural settings and provide valuable information (Culley, Hudson, \& Rapport, 2007). Moreover, the synergistic effects of the group setting elicit ideas and discussion that may not arise in individual interviews (Kidd \& Parshall, 2000). 


\section{Setting}

The present qualitative study was part of a community-based research study being conducted among Brazilian families living in the Greater Boston area, MA, designed to assess Brazilian immigrant mothers' practices of child feeding that may affect the risk of childhood obesity. We worked with three predominantly Brazilian churches (2 Catholic, 1 Protestant) to recruit Brazilian families with preschool-age children (2-5 years old). This study was designed to gain an in-depth understanding of Brazilian immigrant mothers' infant feeding beliefs and practices.

Ethics approval was obtained from the University of Massachusetts Boston Ethics Board (IRB\# 2013060).

\section{Sample}

We recruited a convenience sample of participants through flyers posted at churches, distributed during religious services and at community meetings between January and March 2015. Females were eligible to participate if they were of Brazilian ethnicity, had one or more child between two and five years old, and had been living in the United States for at least 12 months.

\section{Measurement}

Participants completed a brief, self-administered survey after the FGD ended. The survey assessed participants' sociodemographics (e.g., education, marital status, country of origin, length of time living in the United States, etc.), and level of acculturation via the Short Acculturation Scale for Hispanics (SASH) (Marin, Sabogal, Marin, Otero-Sabogal, \& PerezStable, 1987; Marin \& Gamba, 1996). The SASH is a 12-item measuring scale validated for use in Latino groups such as Mexican Americans, Cuban Americans, Puerto Ricans, Dominicans, and Central and South Americans. The SASH assesses language use, media use, and ethnic 
social relations. ${ }^{33,34}$ An acculturation score was computed by averaging across 12 items; measured on a scale of one to five (1=least acculturated, $5=$ fully acculturated) (Marin et al., 1987; Marin \& Gamba, 1996).

\section{Data Collection}

We conducted five FGDs with Brazilian immigrant mothers. FGDs were held at three local churches between March and May 2015. Each FGD had five to seven participants. All five FGDs were conducted in Portuguese, and moderated by one of the authors (ACL), a native Portuguese speaker and the study's principal investigator, trained in qualitative research method. The semi-structured FGD guide explored mothers': (a) beliefs, experiences, and practices with breastfeeding; (b) beliefs and practices related to complementary feeding; (c) sources of information related to infant feeding. The FGD guide was piloted-tested in a FGD with Brazilian immigrant mothers, refined prior to use, and used in all FGDs (see Table 1 for questions used in FGDs). Participants received a $\$ 20.00$ gift card for participation and child care was provided.

Before each FGD, the moderator explained the purpose of the study, procedures, participants' confidentiality of information, and obtained written informed consent from all participants. Before beginning the FGD, the moderator asked participants to think about their youngest preschool-aged children when participating in the discussion. Each audiotaped FGD lasted between 60 and 80 minutes. A trained, bilingual (Portuguese and English) research assistant (RM) took notes during each session. The moderator and research assistant convened for 15 minutes at the end of the FGD in a private room and discussed any new or recurring themes heard during the session, which were entered into a grid of major themes and subthemes. This grid system was used to closely follow the emerging of new themes and subthemes and to determine when data saturation was achieved. 


\section{Data analysis}

We transcribed audiotapes verbatim in Portuguese and analyzed transcripts using thematic analysis (Braun \& Clarke, 2006), an iterative process of coding the data in phases to create meaningful patterns, using the SEM as a framework (Miles \& Huberman, 1994; Pope \& Mays, 1999; Barbour \& Barbour, 2003; Ritchie, Spencer, \& O’Connor, 2004; Braun \& Clarke, 2006; Vaismoradi, Turunen, \& Bondas, 2013). Analytic phases included data familiarization, generation of initial codes, searching for and review of themes and patterns, and defining and naming themes (Braun \& Clarke, 2006; Vaismoradi et al., 2013). Two authors, both nativePortuguese speakers (ACL, MTM) and experienced qualitative researchers, read each transcript several times, then generated initial codes. Items describing a similar idea were coded manually to group common themes. They next reviewed, identified, defined, and named themes (Barbour \& Barbour, 2003; Braun \& Clarke, 2006; Vaismoradi et al., 2013). The two authors responsible for coding met regularly and coded the transcripts independently (Pope \& Mays, 1999; Barbour \& Barbour, 2003; Ritchie, Spencer, \& O’Connor, 2004; Vaismoradi et al., 2013). Inter-rater reliability was calculated using the Cohen's kappa coefficient and found to be $0.73-0.86$ (Pope \& Mays, 1999; Barbour \& Barbour, 2003; Vaismoradi et al., 2013). Minor disagreements in coding were discussed and resolved until consensus was reached (Barbour \& Barbour, 2003).

Descriptive statistics and frequencies were calculated for the sociodemographic data and the SASH scale using Microsoft Excel 2008. The SASH scale has been adapted for use with Brazilian immigrants and research shows good reliability and validity coefficients (alpha coefficient .92) (DeBiaggi, 2001). 


\section{Results}

Five FGDs were conducted before saturation was reached, with no new themes or subthemes emerging during the fifth focus group. Each FGD had an average of six participants (range: 5-7). Participants' $(\mathrm{N}=29)$ ages ranged from 26 to 43 (mean=36.2, $\mathrm{SD}=2.8$ ) years. Approximately $86 \%(\mathrm{n}=25)$ were married with two children on average, and $90 \%(\mathrm{n}=26)$ reported being undocumented U.S. residents. Most $(72 \% ; n=21)$ had completed high school, and $2 \%(\mathrm{n}=6)$ were unemployed. The majority $(92 \% ; \mathrm{n}=21)$ reported being self-employed and owning their own housecleaning business. Approximately half $(52 \% ; n=15)$ reported a family income of $\$ 40,000$ or less. Participants had lived in the United States for an average of 6.4 years $(\mathrm{SD}=2.72)$, and their mean acculturation score was $1.24(\mathrm{SD}=0.59)$, indicating that they identified more closely with Brazilian culture than with American culture.

We classified identified themes into four domains: (1) breastfeeding and complementary feeding beliefs and practices, (2) family and cultural influences and experiences on breastfeeding and complementary feeding, (3) barriers to breastfeeding in the United States, and (4) health programs and healthcare professionals as sources of advice and support. We organized identified themes using the SEM and selected quotes to exemplify identified themes (Table 2).

\section{Individual Influences}

\section{Breastfeeding and complementary feeding beliefs and practices.}

Belief that breastfeeding is beneficial for baby and mother. There was consensus by mothers across all focus groups that breastfeeding was beneficial for both baby and mother. Participants reported several benefits including promoting infant's healthful growth and mother's postpartum weight loss.

Early initiation of breastfeeding. Nearly all mothers reported initiating breastfeeding while at the hospital. Overall, mothers reported a sense of happiness and accomplishment in 
successfully initiating breastfeeding. A few mothers reported having initial difficulties, but spoke of being able to initiate breastfeeding within 24 hours of giving birth with support from nurses and lactation specialists.

Breastfeeding and formula feeding concomitantly is common practice. Nearly all mothers reported breastfeeding and formula feeding their infants concurrently, with most starting this practice while still in the hospital. The majority did not view this practice as conflicting with the recommendation to exclusively breastfeed an infant for the first six months and felt that feeding their infants breast milk and formula at the same time was appropriate. Participants believed that formula feeding ensured that the baby was full, which allowed mother and baby to sleep well. A few mothers also believed that formula had vitamins and nutrients that babies could not get from breast milk. Also, several mothers mentioned using formula when outside the home due to convenience and social norms.

Early interruption of breastfeeding. Although some mothers reported breastfeeding their babies for six months or longer, many reported that they stopped breastfeeding before their babies were six months old. Mothers who stopped breastfeeding before six months, voiced disappointment and stated that it was a hard decision. However, as discussed below, these mothers felt that they needed to stop breastfeeding.

Most mothers who reported discontinuing breastfeeding before six months did so primarily due to work obligations. In addition, some mothers spoke of social pressures, maternal beliefs of insufficient breast milk, and perception of infant hunger and lack of satiety.

Timing of introduction of complementary foods. Most mothers reported introducing their babies to solid foods at around four months due to infant hunger and cues of lack of satiety. In addition, several mothers noted that when they gave their child complementary foods, the 
baby often stopped crying, which mothers interpreted as of confirmation that their child was not satiated by breast milk.

Types of foods commonly introduced. The majority of mothers reported first introducing porridge made with infant cereals, most commonly rice and maize, while several also fed their babies mixed pureed vegetables with or without meat, in addition to porridge. Furthermore, a few mothers reported making their babies' foods from scratch to ensure freshness and that foods were chemical free. Only a few mothers reported buying baby foods for convenience. Those who did this reported using it when outside the home.

\section{Interpersonal Influences}

Family and cultural influences on breastfeeding and introduction of solid foods.

Family and cultural influences. Mothers spoke of sharing their breastfeeding experiences with and receiving advice from relatives. Most mothers felt that their family members, especially their mothers, were a great source of advice about and support for breastfeeding. Mothers also reported the influence of Brazilian food traditions and culture as having a bearing on the timing and types of solid foods they introduced to their babies. Most mothers recalled their own mothers and relatives' infant feeding practices and reported that these practices influenced their own infant feeding practices.

Social norms and cultural influences on breastfeeding. Mothers reported breastfeeding as being the norm in the Brazilian culture, where it is viewed as a natural part of mothers and babies' lives. Furthermore, several mothers spoke of breastfeeding as being easier and less stressful in Brazil due to this cultural acceptance.

The role of healthcare professionals in promoting breastfeeding. Healthcare professionals are important sources of support. Most mothers described receiving information, encouragement, and support for breastfeeding from healthcare 
professionals during their prenatal-care visits and in the hospital after delivery. In addition, some mothers who initially experienced difficulties with breastfeeding reported receiving support from lactation consultants and breastfeeding counselors that enabled them to successfully breastfeed. Home visitations from breastfeeding counselors were seen as important for successful initiation of breastfeeding.

Cultural practices may conflict with advice from healthcare professionals. Mothers reported that information from healthcare professionals about complementary feeding practices, including the appropriate time to introduce solid food and types of foods to introduce often conflicted with cultural beliefs and practices. For example, several mothers reported adding cereal to the baby's bottle to thicken it, often due to their mothers' advice even if this was contrary to what their health care professionals advised. Many mothers reported feeling more comfortable following the advice of their own mothers, other family members and close friends even if advice is counter to practices recommended by healthcare professionals.

\section{Environmental Influences}

\section{Barriers to breastfeeding in the United States.}

Breastfeeding is more difficult in the United States. Mothers perceived that it was more difficult to breastfeed in the United States than in Brazil due to having to prioritize work over breastfeeding, working many and long hours, and lack of maternity leave for self-employed mothers. Mothers also spoke of having less social support from family in the United States than in Brazil and that this lack of support made it difficult to breastfeed because they had to take care not only of their infant, but also older children, household obligations and work.

\section{Organizational Influences}

The role of healthcare programs in promoting breastfeeding. 
Healthcare programs are important sources of support. Several mothers mentioned the Special Supplemental Nutrition Program for Women, Infants, and Children (WIC) being an important source of information and support during pregnancy and after childbirth. Mothers reported that the program was an important source of information related to infant feeding practices including exclusive breastfeeding and appropriate introduction of solid foods during their pregnancies.

\section{Discussion}

This exploratory qualitative study revealed that breastfeeding was widely practiced among Brazilian immigrant mothers. This finding agrees with previous research showing that Latina mothers have high rates of breastfeeding initiation (Bartlick \& Reyes, 2012; Cartagena et al., 2015). However, although most mothers in this study were knowledgeable about the benefits of breastfeeding for both the baby and the mother, our study also revealed that most mothers do not exclusively breastfeed for the first six months of life as recommended. Mothers in our study spoke of routinely feeding their babies both formula and breast milk concomitantly, with most starting this practice within the first few days of their infant's life. Previous studies conducted with Latina women have determined that simultaneously using both formula and breast milk often begins early in the postpartum period due to the maternal belief that combining breast milk and formula feeding provides the best nutrition for the child (Bunik et al., 2006; Bartick \& Reyes, 2012; Cartagena et al., 2015). Feeding both formula and breast milk concomitantly may be associated with decreased breastfeeding duration and early initiation of solid foods, which are practices that are associated with an increased risk of unhealthy weight status and obesity in early childhood and later in life (Pearce et al., 2013; Verstraete et al., 2014; Yan et al., 2014; Cartagena et al., 2015; Lumeng, Taveras, Birch, \& Yanovski, 2015). Future quantitative studies 
should assess the rates of exclusive breastfeeding and the practice of "partial breastfeeding" among Brazilian mothers.

Despite breastfeeding being widely practiced by women in our study, many reported stopping breastfeeding before their children were six months old. Similar to previous studies conducted with Latina women showing that length of time living in the United States is negatively associated with breastfeeding duration, we found that duration of breastfeeding was influenced mainly by work obligations, and to a lesser extent by social pressures, maternal beliefs about insufficient breast milk and of infant hunger (Bunik et al., 2006; Gorman, Madlensky, Jackson, Ganiats, \& Boies, 2007; Harley, Stamm, \& Eskenazi, 2007; Choudhry \& Wallace, 2012; Gibbs \& Forste, 2014; Glassman, McKearney, Saslaw, \& Sirota, 2014; Hawley et al., 2015). In addition, a few mothers in our study reported that delivery nurses offered them the option of introducing formula to their infants while in the hospital to allow mother and baby to sleep better. Many U.S. maternity care practices are not compliant with the Ten Steps to Successful Breastfeeding, a hospital-based set of practices shown to support breastfeeding both collectively and individually, and may influence poor breastfeeding practices, including short breastfeeding duration (UNICEF \& World Health Organization, 1989; Saadeh \& Akre, 1996; Chien, Tai, Chu, Ko, \& Chiu, 2007; World Health Organization \& UNICEF, 2009; Nickel, Labbok, Hudgens, \& Daniels, 2012).

Mothers participating in our study believed that feeding their babies formula ensured that infant was fully sated and "slept well." This finding suggests that mothers' may not understand infant's ability to self-regulate food intake. This maternal feeding practice may lead to infant overfeeding, which has been shown to be associated with increased risk of child obesity. ${ }^{16}$ In addition, Brazilian mothers may practice non-exclusive breastfeeding due to the belief that a combination of breast milk and formula is best for the infant's health and satiety. This belief may 
be reinforced by the receipt of free formula in hospitals at childbirth and postpartum discharge and by WIC agencies following childbirth. This finding points to potential influence of the WIC program on mothers' infant feeding practices. Previous studies with low-income, Hispanic mothers enrolled in the WIC program have shown that WIC participants have inadequate breastfeeding practices including early supplementation of formula (Bunik et al., 2006; Bartick \& Reyes, 2012; Cartagena et al., 2015). Future studies should examine and quantify the effect of WIC participation on Brazilian mothers' breastfeeding practices.

Family and culture emerged as important influences on mothers' infant-feeding beliefs and practices. This finding is important since there is a growing body of literature linking obesogenic infant feeding practices such as introducing solid foods early and adding cereal to the bottle to increased risk of unhealthy weight status and obesity (Anzman, Rollins, \& Birch, 2010; Dattilo et al., 2012; Davis, Whaley, \& Goran, 2012; Moss \& Yeaton, 2014; Verstraete et al., 2014; Lumeng et al., 2015). Our study also revealed that mothers might defer to the advice and cultural beliefs of their families over advice offered by healthcare providers. This finding indicates that breastfeeding promotion interventions designed for Brazilian immigrant mothers should recognize cultural beliefs and delayed introduction of solids within the context of family and cultural traditions. In addition, interventions should consider targeting physicians and other healthcare providers to ensure they offer proper guidance to breastfeeding mothers that are family-centered and culturally sensitive.

Future studies are needed to further explore and understand multiple influences on Brazilian immigrant mothers' infant feeding decisions and practices and to quantify factors influencing these practices as well as the effects of these practices on child's weight status in infancy and early childhood. This information will be important to identify factors among this ethnic group amenable to intervention. 
To our knowledge, this is the first study to explore infant feeding practices of immigrant Brazilian mothers living in the United States. Although breastfeeding is common practice in Brazil and duration is often longer than six months, many Brazilian immigrant mothers in the United States may need to prioritize work over breastfeeding. Furthermore, lack of maternity leave for self-employed mothers make maintaining breastfeeding difficult. Innovative strategies to promote continued breastfeeding among Brazilian immigrant mothers in the United States are needed. Additional research would be well poised to explore the relationship between immigrant status, self-employment and breastfeeding duration among Brazilian immigrant mothers in the United States. This information will be important for the development of effective breastfeeding promotion strategies targeting immigrant Brazilian mothers.

Study findings should be viewed in light of limitations, including limited transferability due to purposeful sampling, a small sample recruited from religious organizations, and participants possibly having a heightened interest and awareness regarding the topics. Furthermore, given that children's age ranged between two and five years at the time of the study, mothers' recollection of infant feeding practices may have been affected by recall bias. Another study limitation is the lack of quantitative data on rates of breastfeeding initiation and duration of mothers participating in the study. The lack of this information makes it impossible to assess potential selection bias. Future research can address study limitations by employing quantitative methods, recruiting from other community-based organizations, and exploring immigrant Brazilian mothers' beliefs and practices related to infant feeding from other communities across the United States. Nevertheless, this qualitative study provided deeper insight into immigrant Brazilian mothers' personal beliefs and practices related to infant feeding. 


\section{Conclusions}

The present study provides new information on Brazilian immigrant mothers' beliefs and infant feeding practices that may provide important targets for interventions aimed at promoting healthful infant feeding practices and decreasing disparities in early childhood obesity among Brazilian children of immigrant families. To our knowledge, this is the first study to explore infant feeding practices of Brazilian immigrant mothers living in the United States. Innovative strategies such as interventions providing anticipatory guidance and incorporating technologyfacilitated education and consultations to offset early termination of breastfeeding among Brazilian immigrant mothers in the United States are need (Wen et al., 2007; Paul et al., 2014; Daniels et al., 2015; Friesen, Hormuth, Petersen, \& Babbitt, 2015). Additional research is needed to quantify and provide further understanding of the relationship between immigrant status, selfemployment and breastfeeding duration among Brazilian immigrant mothers in the United States. This information will allow for the development of efficacious intervention designed to promote and sustain breastfeeding by Brazilian immigrant mothers. Furthermore, effective interventions will be those that incorporate an understanding of the socio-economic, family, and cultural influences on infant feeding practices and develop health promotion messages tailored to the specific needs of this minority group.

\section{Funding}

The authors received no financial support for the research, authorship, and/or publication of this article.

\section{Declaration of Conflicting Interests}

The authors declared no potential conflicts of interest with respect to the research, authorship, and/or publication of this article. 


\section{Supplementary materials}

Data and all other materials for this study are kept at the Department of Exercise and Health Sciences, University of Massachusetts Boston. The datasets generated during and/or analyzed during the current study are not publicly available due the terms of consent to which the participants agreed but are available from the corresponding author on reasonable request. 


\section{References}

American Academy of Pediatrics. (2012). Policy statement: Breastfeeding and the use of human milk. Pediatrics, 129(3), e827-e841.

Anzman, S. L., Rollins, B. Y., \& Birch, L. L. (2010). Parental influence on children's early eating environments and obesity risk: implications for prevention. International Journal of Obesity, 34(7), 1116-1124.

Baker, J. L, Michaelsen, K. F., Rasmussen, K. M., \& Sorensen, T. I. (2004). Maternal prepregnant body mass index, duration of breastfeeding, and timing of complementary food introduction are associated with infant weight gain. American Journal of Clinical Nutrition, 80(6), 1579-88.

Barbour, R. S., \& Barbour, M. (2003). Evaluating and synthesizing qualitative research: the need to develop a distinctive approach. Journal of Evaluation in Clinical Practice, 9(2), 179186.

Bartick, M., \& Reyes, C. (2012). Las dos cosas: an analysis of attitudes of latina women on nonexclusive breastfeeding. Breastfeeding Medicine, 7(1), 19-24.

Brasil, Ministério da Saúde, Centro Brasileiro de Análise e Planejamento. (2009). Pesquisa Nacional de Demografia e Saúde da Criança e da Mulher-PNDS 2006: Relatório final. Brasília: Ministério da Saúde; 2009.

Braun, V., \& Clarke, V. (2006). Using thematic analysis in psychology. Qualitative Research in Psychology, 3(2), 77-101.

Bunik, M., Clark, L., Zimmer, L. M., Jimenez, L. M., O'Connor, M. E., Crane, L. A., \& Kempe, A. (2006). Early infant feeding decisions in low-income Latinas. Breastfeeding Medicine, l(4), 225-235. 
Cartagena, D., Ameringer, S. W., McGrath, J. M., Masho, S. W., Jallo, N., \& Myers, B. J. (2015). Factors contributing to infant overfeeding in low-income immigrant Latina mothers. Applied Nursing Research, 28(4), 316-321. doi:10.1016/j.apnr.2015.03.007.

Chien, L. Y., Tai, C. J., Chu, K. H., Ko, Y. L., \& Chiu, Y. C. (2007). The number of Baby Friendly hospital practices experienced by mothers is positively associated with breastfeeding: a questionnaire survey. International Journal of Nursing Studies, 44(7), $1138-1146$.

Choudhry, K., \& Wallace, L. M. (2012). 'Breast is not always best': South Asian women's experiences of infant feeding in the UK within an acculturation framework. Maternal \& Child Nutrition, 8(1), 72-87. doi:10.1111/j.1740-8709.2010.00253.x.

Culley, L., Hudson, N., \& Rapport, F. (2007). Using focus groups with minority ethnic communities: Researching infertility in British South Asian communities. Qualitative Health Research, 17(1), 102-112.

Daniels, L. A., Mallan, K. M., Nicholson, J. M., Thorpe, K., Nambiar, S., Mauch, C. E., \& Magarey, A. (2015). An early feeding practices intervention for obesity prevention. Pediatrics, 136(1), e40-e49.9. doi:10.1542/peds.2014-4108.

Dattilo, A. M., Birch, L., Krebs, N. F., Lake, A., Taveras, E. M., \& Saavedra, J. M. (2012). Need for early interventions in the prevention of pediatric overweight: a review and upcoming directions. Journal of Obesity, 2012. doi:10.1155/2012/123023.

Davis, J. N., Whaley, S. E., \& Goran, M. I. (2012). Effects of breastfeeding and low sugarsweetened beverage intake on obesity prevalence in Hispanic toddlers. The American Journal of Clinical Nutrition, 95(1), 3-8.

DeBiaggi, S.D.D. (2001). Changing Gender Roles: Brazilian Immigrant Families in the U.S. E1 Paso, TX: LFB Scholarly Publishing LLC. 
Dixon, B., Peña, M. M., \& Taveras, E. M. (2012). Lifecourse approach to racial/ethnic disparities in childhood obesity. Advances in Nutrition: An International Review Journal, 3(1), 73-82. doi:10.3945/an.111.000919. doi:10.1001/jamapediatrics.2014.3554.

Ebenegger, V., Marques-Vidal, P. M., Nydegger, A., Laimbacher, J., Niederer, I., Bürgi, F., ... \& Puder, J. J. (2011). Independent contribution of parental migrant status and educational level to adiposity and eating habits in preschool children. European Journal of Clinical Nutrition, 65(2), 210-218. doi: 10.1038/ejcn.2010.248.

Fewtrell, M. S., Haschke, F., \& Prescott, S. L. (Eds). (2014). Preventive Aspects of Early Nutrition. 85th Nestle Nutrition Institute Workshop Series. London:Karger AG Basel. doi:10.1159/000439501.

Friesen, C. A., Hormuth, L. J., Petersen, D., \& Babbitt, T. (2015). Using Videoconferencing Technology to Provide Breastfeeding Support to Low-Income Women Connecting Hospital-Based Lactation Consultants with Clients Receiving Care at a Community Health Center. Journal of Human Lactation, 31(4), 595-599. doi:

10.1177/0890334415601088.

Gibbs, B. G., \& Forste, R. (2014). Socioeconomic status, infant feeding practices and early childhood obesity. Pediatric Obesity, 9(2), 135-146. doi:10.1111/j.20476310.2013.00155.x.

Glassman, M. E., McKearney, K., Saslaw, M., \& Sirota, D. R. (2014). Impact of breastfeeding self-efficacy and sociocultural factors on early breastfeeding in an Urban, Predominantly Dominican Community. Breastfeeding Medicine, 9(6), 301-307. doi:10.1089/bfm.2014.0015.

Godfrey, B. J., Jouët-Pastré, C., \& Braga, L. J. (2009). Becoming Brazuca: Brazilian Immigration to the United States. Cambridge, Mass.: Harvard University Press. 
Gorman, J. R., Madlensky, L., Jackson, D. J., Ganiats, T. G., \& Boies, E. (2007). Early postpartum breastfeeding and acculturation among Hispanic women. Birth, 34(4), 308315.

Gross, R. S., Mendelsohn, A. L., Fierman, A. H., Hauser, N. R., \& Messito, M. J. (2014). Maternal infant feeding behaviors and disparities in early child obesity. Childhood Obesity, 10(2), 145-152.

Harley, K., Stamm, N. L., \& Eskenazi, B. (2007). The effect of time in the US on the duration of breastfeeding in women of Mexican descent. Maternal and Child Health Journal, 11(2), $119-125$.

Hawley, N. L., Rosen, R. K., Strait, E. A., Raffucci, G., Holmdahl, I., Freeman, J. R., ... \& McGarvey, S. T. (2015). Mothers' attitudes and beliefs about infant feeding highlight barriers to exclusive breastfeeding in American Samoa. Women and Birth, 28(3), e80e86. doi:10.1016/j.wombi.2015.04.002. Epub 2015 Apr 29.

Institute of Medicine, Food and Nutrition Board. (2015, September). Examining a developmental approach to childhood obesity: The fetal and early childhood years - workshop summary. Washington, DC: National Academies Press.

Institute of Medicine. (2011). Early childhood obesity prevention policies. Washington, DC: The National Academies Press.

Kidd, P. S., \& Parshall, M. B. (2000). Getting the focus and the group: enhancing analytical rigor in focus group research. Qualitative Health Research, 10(3), 293-308.

Kimbro, R. T., Brooks-Gunn, J., \& McLanahan, S. (2007). Racial and ethnic differentials in overweight and obesity among 3-year-old children. American Journal of Public Health, $97(2), 298-305$. 
Lima, A., \& Siqueira, C. E. (2007). Brazilians in the US and Massachusetts: A demographic and economic profile. Retrieved January 16, 2013, from http://scholarworks.umb.edu.ezproxy.lib.umb.edu/gaston_pubs/50.

Lumeng, J. C., Taveras, E. M., Birch, L., \& Yanovski, S. Z. (2015). Prevention of obesity in infancy and early childhood: a National Institutes of Health workshop. JAMA Pediatrics, 169(5), 484-90.Marín, G., \& Gamba, R. J. (1996). A new measurement of acculturation for Hispanics: The Bidimensional Acculturation Scale for Hispanics (BAS). Hispanic Journal of Behavioral Sciences, 18(3), 297-316.

Marin, G., Sabogal, F., Marin, B. V., Otero-Sabogal, R., \& Perez-Stable, E. J. (1987). Development of a short acculturation scale for Hispanics. Hispanic Journal of Behavioral Sciences, 9(2), 183-205.

McLeroy, K. R., Bibeau, D., Steckler, A., \& Glanz, K. (1988). An ecological perspective on health promotion programs. Health Education \& Behavior, 15(4), 351-377.

Miles M., \& Huberman A. 1994. Qualitative data analysis, 2nd ed. Thousand Oaks: Sage Publications.

Moss, B. G., \& Yeaton, W. H. (2014). Early childhood healthy and obese weight status: potentially protective benefits of breastfeeding and delaying solid foods. Maternal and Child Health Journal, 18(5), 1224-1232.. doi:10.1007/s10995-013-1357-z.

Nickel, N. C., Labbok, M. H., Hudgens, M. G., \& Daniels, J. L. (2012). The extent that noncompliance with the ten steps to successful breastfeeding influences breastfeeding duration. Journal of Human Lactation, 0890334412464695. doi: $10.1177 / 0890334412464695$. 
Ogden, C. L., Carroll, M. D., Kit, B. K., \& Flegal, K. M. (2014). Prevalence of childhood and adult obesity in the United States, 2011-2012. JAMA, 311(8), 806-814. doi:10.1001/jama.2014.732.

Pan, L., Grummer-Strawn, L. M., McGuire, L. C., Park, S., \& Blanck, H. M. (2015). Trends in state/territorial obesity prevalence by race/ethnicity among US low-income, preschoolaged children. Pediatric Obesity. doi:10.1111/ijpo.12078.

Paul, I. M., Williams, J. S., Anzman-Frasca, S., Beiler, J. S., Makova, K. D., Marini, M. E., ... \& Birch, L. L. (2014). The intervention nurses start infants growing on healthy trajectories (INSIGHT) study. BMC Pediatrics, 14(1), 1. doi: 10.1186/1471-2431-14-184.

Pearce, J., Taylor, M. A., \& Langley-Evans, S. C. (2013). Timing of the introduction of complementary feeding and risk of childhood obesity: a systematic review. International Journal of Obesity, 37(10), 1295-1306. doi:10.1038/ijo.2013.99.

Pope C., Ziebland, S., \& Mays, N. (1999). Analysing qualitative data. In C. Pope \& N. Mays N (Eds.), Qualitative research in health care, (2nd ed., pp. 75-88). London: BMJ Books.

Ritchie, J., Spencer, L., \& O'Connor, W. 2004. Carrying out qualitative analysis. In J. Ritchie \& J. Lewis (Eds.), Qualitative research practice (pp. 219-262). London: Sage Publications.

Saadeh R., \& Akré, J. (1996).Ten steps to successful breastfeeding: a summary of the rationale and scientific evidence. Birth, 23(3), 154-60.

Sanchez-Vaznaugh, E. V., Kawachi, I., Subramanian, S. V., Sánchez, B. N., \& Acevedo-Garcia, D. (2008). Differential effect of birthplace and length of residence on body mass index (BMI) by education, gender and race/ethnicity. Social Science \& Medicine, 67(8), 13001310. doi: 10.1016/j.socscimed.2008.06.015.

Singh, G. K., Siahpush, M., Hiatt, R. A., \& Timsina, L. R. (2011). Dramatic increases in obesity and overweight prevalence and body mass index among ethnic-immigrant and social 
class groups in the United States, 1976-2008. Journal of Community Health, 36(1), 94110. doi:10.1007/s10900-010-9287-9.

Sussner, K. M., Lindsay, A. C., Peterson, K. E. (2008). The influence of acculturation on breastfeeding initiation and duration in low-income women in the US. Journal of Biosocial Science, 40(5), 673-96.

Thompson, A. L., \& Bentley, M. E. (2013). The critical period of infant feeding for the development of early disparities in obesity. Social Science \& Medicine, 97, 288-296. doi:10.1016/j.socscimed.2012.12.007.

Tovar, A., Hennessy, E., Must, A., Hughes, S. O., Gute, D. M., Sliwa, S., ... \& Pirie, A. (2013). Feeding styles and evening family meals among recent immigrants. International Journal of Behavioral Nutrition and Physical Activity, 10(1), 1. doi:10.1186/1479-5868-10-84.

UNICEF, \& World Health Organization. (1989). Protecting, promoting and supporting breastfeeding: the special role of maternity services. Geneva: World Health Organization.

Vaismoradi, M., Turunen, H., \& Bondas, T. (2013). Content analysis and thematic analysis:

Implications for conducting a qualitative descriptive study. Nursing \& Health Sciences, 15(3), 398-405. doi:10.1111/nhs.12048

Venancio, S. I., Escuder, M. M., Saldiva, S. R., \& Giugliani, E. R. (2010). Breastfeeding practice in the Brazilian capital cities and the Federal District: Current status and advances. Journal of Pediatrics (Rio J),86(4), 317-24. doi:10.2223/JPED.2016.

Verstraete, S. G., Heyman, M. B., Wojcicki, J. M. (2014). Breastfeeding offers protection against obesity in children of recently immigrated Latina women. Journal of Community Health, 39(3), 480-6. doi:10.1007/s10900-013-9781-y.

Vieira, T. O., Vieira, G. O., de Oliveira, N. F., Mendes, C. M. C., Giugliani, E. R. J., \& Silva, L. R. (2014). Duration of exclusive breastfeeding in a Brazilian population: new 
determinants in a cohort study. BMC Pregnancy Childbirth, 14, 175. doi:10.1186/14712393-14-175.

Wen, L. M., Baur, L. A., Rissel, C., Wardle, K., Alperstein, G., \& Simpson, J. M. (2007). Early intervention of multiple home visits to prevent childhood obesity in a disadvantaged population: a home-based randomised controlled trial (Healthy Beginnings Trial). $B M C$ Public Health, 7(1), 1.

Weng, S. F., Redsell, S. A., Swift, J. A., Yang, M., \& Glazebrook, C. P. (2012). Systematic review and meta-analyses of risk factors for childhood overweight identifiable during infancy. Archives of Disease in Childhood, 97(12), 1019-1026. doi:10.1136/archdischild2012-302263.

World Health Organization, \& UNICEF. (2009). Baby-friendly hospital initiative: revised, updated and expanded for integrated care. http://www.who.int.ezproxy.lib.umb.edu /nutrition/publications/infantfeeding/9789241594950/en/index.html. Accessed February $14,2015$.

World Health Organization. (2008). Indicators for assessing infant and young child feeding practices: Conclusions of a consensus meeting held 6-8 November 2007 in Washington, $D C, U S A$. Geneva: World Health Organization.

World Health Organization. (2014). Report of the first meeting of the ad hoc working group on science and evidence for ending childhood obesity. Geneva: World Health Organization.

Yan, J., Liu, L., Zhu, Y., Huang, G., \& Wang, P. P. (2014). The association between breastfeeding and childhood obesity: a meta-analysis. BMC Public Health, 14(1), 1. 